\title{
Pristimerin demonstrates anticancer potential in colorectal cancer cells by inducing G1 phase arrest and apoptosis and suppressing various pro-survival signaling proteins
}

\author{
BASHIR A. YOUSEF ${ }^{1,3}$, MOUNIA GUERRAM ${ }^{1}$, HOZEIFA M. HASSAN ${ }^{1,4}$, AIDA M. HAMDI ${ }^{1}$, \\ LU-YONG ZHANG ${ }^{1,2}$ and ZHEN-ZHOU JIANG ${ }^{1,2}$ \\ ${ }^{1}$ Jiangsu Key Laboratory of Drug Screening, and ${ }^{2}$ Jiangsu Center for Pharmacodynamics Research and Evaluation, \\ China Pharmaceutical University, Nanjing, Jiangsu 210009, P.R. China; ${ }^{3}$ Department of Pharmacology, \\ Faculty of Pharmacy, University of Khartoum, 1996 Khartoum; ${ }^{4}$ Department of Pharmacology, \\ Faculty of Pharmacy, University of Gezira, Wad Medani, Sudan
}

Received August 4, 2015; Accepted September 28, 2015

DOI: $10.3892 /$ or.2015.4457

\begin{abstract}
Pristimerin is a naturally occurring triterpenoid that has a cytotoxic effect on several cancer cell lines. However, the cytotoxic effects of pristimerin as well as its molecular mechanisms of action against colorectal cancer have never been explored. In the present study, we investigated the anticancer potential of pristimerin, and examined the different signaling pathways affected by its action in three colon cancer cell lines namely HCT-116, COLO-205 and SW-620. Pristimerin was found to possess potent cytotoxic and proliferation inhibitory effects against these cell lines. Cell cycle analysis revealed G1 phase arrest, which was strongly associated with decreased expression of cyclin D1 and cyclin-dependent kinases (cdk4 and cdk6) with concomitant induction of p21. Pristimerin also induced apoptosis in a dose-dependent manner. Cell plasma membrane alterations studied by Annexin V/PI double staining, loss of mitochondrial membrane potential $(\Delta \Psi \mathrm{m})$, measurements of caspase activities and the inhibitory effect of Z-VAD-FMK (a caspase inhibitor) confirmed the apoptotic effect of pristimerin. Moreover, western blot data showed that apoptotic induction was associated with activated caspase-3 and -8, PARP-1 cleavage and modulation of the expression levels of Bcl-2 family proteins. Additionally, pristimerin treatment downregulated the phosphorylated forms of EGFR and HER2 proteins, and subsequently caused a decrease in the phosphorylated forms of Erk1/2, Akt, mTOR and NF- $\mathrm{B}$
\end{abstract}

Correspondence to: Professor Lu-Yong Zhang or Dr Zhen-Zhou Jiang, Jiangsu Key Laboratory of Drug Screening, China Pharmaceutical University, 24 Tongjiaxiang Street, Nanjing, Jiangsu 210009, P.R. China

E-mail: lyzhang@cpu.edu.cn

E-mail: beaglejiang@cpu.edu.cn

Key words: pristimerin, colorectal cancer, G1 phase arrest, apoptosis, pro-survival signaling proteins proteins. Taken together, these results suggest that pristimerin may have potential as a new targeting therapeutic strategy for the treatment of colon cancer.

\section{Introduction}

Colorectal cancer (CRC) is the third most common cancer worldwide, with more than 1 million new cases diagnosed each year (1). CRC is the fourth most common cause of cancerrelated mortality worldwide (1), and the third most common cause of cancer-related mortality in the US (2). CRC is a heterogeneous disease with different molecular characteristics such as abnormal genetic and epigenetic alterations in several signaling pathways which are responsible for controlling cell proliferation, apoptosis and invasion of CRC $(3,4)$. The management of CRC has improved in the last decade, in term of screening, surgical management and adjuvant chemotherapy.

Natural compounds have become important in anticancer drug development as they are more tolerable to the human body (5). Pristimerin (Fig. 1A) is a naturally occurring quinonemethide triterpenoid compound isolated from the Celastraceae and Hippocrateaceae families. Pristimerin has long been used as an anti-inflammatory, antioxidant, antimalarial and insecticidal agent $(6,7)$. It also has promising clinical potential as both a chemotherapeutic and chemopreventive agent for cancer. Indeed, it has been reported to exhibit a cytotoxic effect on several human cancer cell lines including breast (8), pancreatic (9), hepatic (10), cervical (11) and prostate cancer (12), and glioma tumor cells (13). However, the exact mechanism of pristimerin cytotoxicity has not been completely explored. Furthermore, pristimerin has been reported to induce cancer cell death via different mechanisms of action, including the caspase-dependent apoptotic cascade (8), potent proteasomal inhibition (14), suppression of nuclear factor- $\kappa \mathrm{B}$ $(\mathrm{NF}-\kappa \mathrm{B})$ activity (15), and oxidative stress induction (11).

Although pristimerin has a variety of reported antitumor activities, its effect on colon cancer cell lines and the underlying mechanism of action have never been explored. In the present study, we examined the effect of pristimerin on three 
human colon cancer cell lines namely HCT-116, SW-620 and COLO-205. Our results revealed that pristimerin strongly suppressed the growth of these cell lines by inducing G1 phase cell cycle arrest and apoptosis. Furthermore, the anticancer effects of pristimerin were associated with inhibition of EGFR and HER 2 and related downstream signaling pathways.

\section{Materials and methods}

Materials. Pristimerin with a purity of $>97 \%$ was purchased from Enzo Life Sciences (Lausen, Switzerland). 3-(4,5-Dimethylthiazol-2-yl)-2,5-diphenyltetrazolium bromide (MTT) was purchased from Sigma (St. Louis, MO, USA). Annexin V-FITC apoptosis detection kit was purchased from BD Pharmingen (San Diego, CA, USA). Cell cycle assay kit, caspase-3, -8 and -9 activity assay kits and mitochondrial membrane potential $\left(\Delta \Psi_{\mathrm{m}}\right)$ assay kit were purchased from Beyotime Institute of Biotechnology (Nanjing, China). Antibodies against cleaved caspase-3, cleaved caspase-8, Bcl-2, Bcl-xL, Bax, PARP-1, HER2, p-HER2, p-EGFR, mTOR, p-mTOR, cyclin D1, Cdk-4, cdk-6, Rb and p-Rb were obtained from Cell Signaling Technology (Danvers, MA, USA). Antibodies against ERK1/2, p-ERK1/2, Akt, p-Akt,

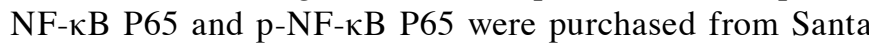
Cruz Biotechnology (Santa Cruz, CA, USA). The antibody against EGFR was purchased from Abcam (Cambridge, $\mathrm{UK}$ ); both anti-mouse IgG-horseradish peroxidase, anti-goat IgG-horseradish peroxidase and anti-rabbit IgG-horseradish peroxidase were purchased from KangChen Biotechnology (Shanghai, China).

Cell lines and cell culture. The human colon cancer cell lines HCT-116, SW-620 and COLO-205 were obtained from the American Type Culture Collection (ATCC; Manassas, VA, USA). The HCT-116 and COLO-205 cells were cultured in RPMI-1640 medium (HyClone China Ltd., China), supplemented with $10 \%$ fetal bovine serum (FBS; Gibco-BRL, Grand Island, NY, USA), penicillin (100 U/ml) and streptomycin $(100 \mu \mathrm{g} / \mathrm{ml})$ (Sigma) and maintained at $37^{\circ} \mathrm{C}$ in a humidified atmosphere containing $5 \% \mathrm{CO}_{2}$. SW-620 cells were cultured in Leibovitz's L-15 medium (HyClone China Ltd.), supplemented with $10 \% \mathrm{FBS}$, penicillin $(100 \mathrm{U} / \mathrm{ml})$ and streptomycin $(100 \mu \mathrm{g} / \mathrm{ml})$ at $37^{\circ} \mathrm{C}$ under a humidified $100 \%$ atmosphere.

Cell viability assay. The inhibitory effect of pristimerin on cell viability was measured by MTT method. Briefly, $5 \times 10^{3}$ cells/well were seeded into 96 -well plates for $24 \mathrm{~h}$, and then the medium was replaced with fresh medium containing various concentrations of pristimerin $(0,0.1,0.5,1,5$ and $10 \mu \mathrm{M}$ ) for $12,24,48$ and $72 \mathrm{~h}$. After incubation, MTT was added to each well at a final concentration of $0.5 \mathrm{mg} / \mathrm{ml}$. The insoluble formazan was collected and dissolved in dimethylsulfoxide (DMSO) and measured with a microplate reader (Safire²; Tecan, Switzerland) at $540 \mathrm{~nm}$.

DNA cell cycle analysis. Cells were treated with various pristimerin concentrations $(0,0.5,1$ and $2 \mu \mathrm{M})$ in complete medium for $48 \mathrm{~h}$. Both floating and adherent cells were harvested by centrifugation (3,000 $\mathrm{g}$ for $5 \mathrm{~min}$ ), washed twice with ice-cold phosphate-buffered saline (PBS) and fixed in ice-cold $75 \%$ ethanol at $4^{\circ} \mathrm{C}$ overnight. The ethanol was subsequently removed after centrifugation, and $\sim 1 \times 10^{6}$ cells were re-suspended in PBS containing $25 \mu \mathrm{g} / \mathrm{ml}$ RNase. Samples were then incubated with propidium iodide (PI) $(50 \mu \mathrm{g} / \mathrm{ml})$ at $37^{\circ} \mathrm{C}$ for $30 \mathrm{~min}$ and were analyzed by a flow cytometer (Becton-Dickinson, San Jose, CA, USA).

Annexin V/PI double-staining assay. Apoptosis was measured by a flow cytometer using Annexin V/PI double staining. HCT-116, SW-620 and COLO-205 cells were cultured in a $60-\mathrm{mm}$ dish at a density of $2 \times 10^{5} / \mathrm{dish}$. On the following day, the cells were treated with pristimerin $(0,0.5,1$ and $2 \mu \mathrm{M})$. After $48 \mathrm{~h}$, the cells were harvested, both floating and attached cells were collected, washed with ice-cold PBS twice, and re-suspended in $200 \mu \mathrm{l}$ binding buffer containing Annexin V and $40 \mathrm{ng} / \mathrm{sample} \mathrm{PI}$ for $15 \mathrm{~min}$ at $37^{\circ} \mathrm{C}$ in the dark (according to the manufacturer's instructions). The number of viable, apoptotic and necrotic cells was then quantified by a flow cytometer.

Analysis of caspase-3, -8 and -9 activities. Caspase-3,-8 and -9 activities were measured using colometric assay kits as previously described (16) and following the manufacturer's guideline. Briefly, HCT-116, SW-620 and COLO-205 cells without or with $(0.5,1$ and $2 \mu \mathrm{M})$ pristimerin treatment, were washed with cold PBS, re-suspended in lysis buffer and left on ice for $15 \mathrm{~min}$. The lysate was centrifuged at $16,000 \mathrm{x} \mathrm{g}$ at $4^{\circ} \mathrm{C}$ for 15 min. Activities of caspase-3, -8 and -9 were measured using substrate peptides Ac-DEVD-pNA, Ac-IETD-pNA and Ac-LEHD-pNA, respectively. Release of p-nitroanilide (pNA) was qualified by determining the absorbance with a spectrophotometer. Data were normalized against the control untreated cells.

Pan-caspase inhibitor Z-VAD-FMK. HCT-116, SW-620 and COLO-205 cells were seeded into 96-well plates at an initial density of $5 \times 10^{3}$ cells/well. After $24 \mathrm{~h}$, the cells were pre-incubated with $50 \mu \mathrm{M}$ Z-VAD-FMK (Beyotime Institute of Biotechnology) for $1 \mathrm{~h}$ and then incubated with pristimerin $(0,0.5,1,2,4$ and $10 \mu \mathrm{M})$ for $48 \mathrm{~h}$. The inhibition rate $(\%)$ was measured by MTT in both the presence and absence of Z-VAD-FMK.

$\Delta \Psi m$ assay. The JC-1 probe was used to measure mitochondrial depolarization in HCT-116, SW-620 and COLO-205 cells. Briefly, the cells were cultured into 6-well plates; after treatment with pristimerin $(0,0.5,1$ and $2 \mu \mathrm{M})$ for $48 \mathrm{~h}$, they were incubated with an equal volume of a JC-1 staining solution $(5 \mu \mathrm{g} / \mathrm{ml})$ at $37^{\circ} \mathrm{C}$ for $20 \mathrm{~min}$ and rinsed twice with PBS. Changes in $\Delta \Psi \mathrm{m}$ were analyzed using a flow cytometer with the excitation and emission wavelengths of 488 and $525 \mathrm{~nm}$, respectively.

Whole-cell lysates and western blot analysis. HCT-116, SW-620 and COLO-205 cells were collected following treatment and lysed by lysis buffer (KangChen Biotechnology) for $30 \mathrm{~min}$ on ice. Cell lysates were centrifuged at $12,000 \mathrm{rpm}$ for $15 \mathrm{~min}$ at $4^{\circ} \mathrm{C}$. Total protein concentration in the supernatants was detected by a bicinchoninic acid (BCA) protein assay kit (Beyotime Institute of Biotechnology). Equal amounts of lysate proteins were separated on 8-12\% SDS-PAGE and transferred 
B

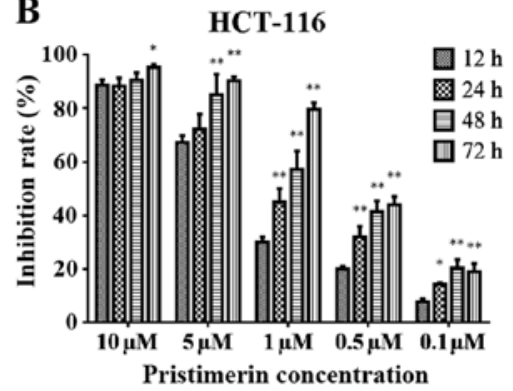

A

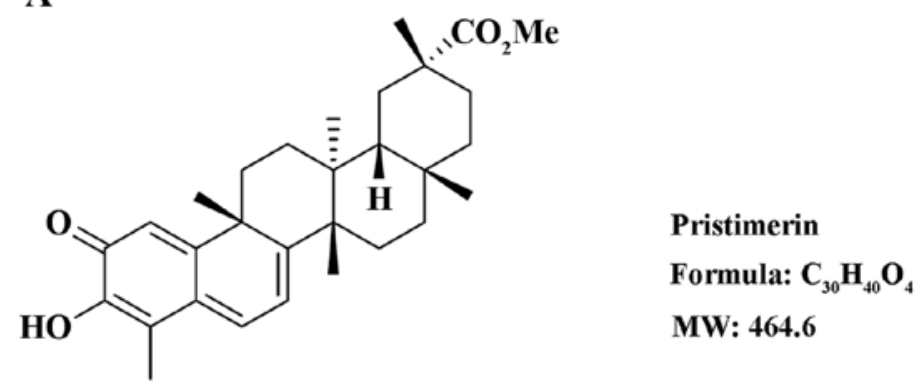

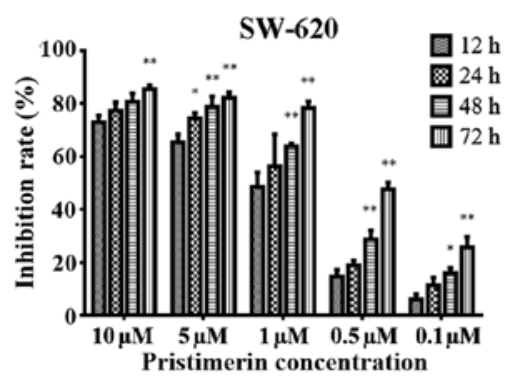

C
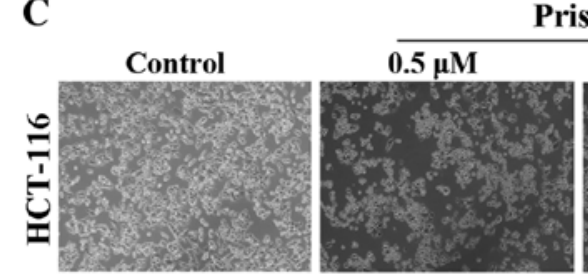

Pristimerin concentration
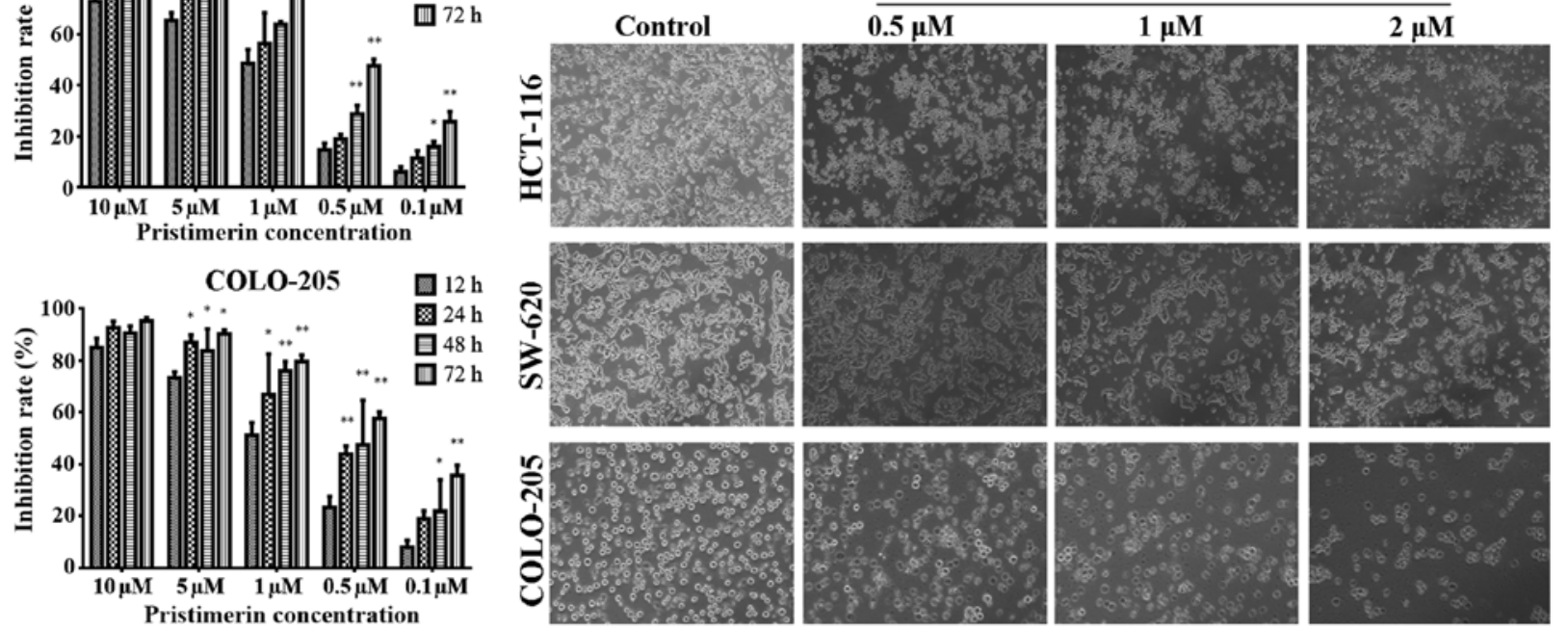

Figure 1. Pristimerin inhibits colon cancer cell growth. (A) Chemical structure of pristimerin. (B) HCT-116, SW-620 and COLO-205 cells were grown in the absence and presence of different concentrations of pristimerin for 12, 24, 48 and $72 \mathrm{~h}$. Cell viability was measured by MTT assay. Inhibition rates (\%) were presented as mean \pm SD from three independent experiments. ${ }^{*} \mathrm{p}<0.05,{ }^{* *} \mathrm{p}<0.01 \mathrm{vs} .12 \mathrm{~h}$ of pristimerin treatment. (C) Morphological changes in cell cultures visualized by light microscopy $48 \mathrm{~h}$ after treatment with pristimerin. Each experiment was repeated three times.

onto nitrocellulose membranes. The non-specific binding sites were blocked with TBST buffer containing $5 \%$ free-fat milk for $2 \mathrm{~h}$. Following overnight incubation at $4^{\circ} \mathrm{C}$ with specific primary antibodies, the membranes were washed three times with TBST buffer and incubated at room temperature for $1 \mathrm{~h}$ with horseradish peroxidase-conjugated secondary antibody. After three washes with TBST buffer, the immunoblots were visualized using the enhanced chemiluminescence (ECL; Millipore Corporation) detection kit. Immunoreactive bands were scanned (GS-800) and analyzed using a ChemiDoc XRS imaging system (Quantity One, v4.4.0) (both from Bio-Rad Life Sciences, Hercules, CA, USA).

Statistical analysis. Data were analyzed using GraphPad Prism 6 (GraphPad Software, Inc., San Diego, CA, USA). All data are expressed as mean \pm standard deviation (SD) from at least three independent experiments. The statistical comparisons between the control and treatment groups were determined using the Student's two-tailed t-test. Differences with $\mathrm{p}<0.05$ were considered statistically significant.

\section{Results}

Pristimerin exerts potent cytotoxicity against colon cancer cells. Pristimerin cytotoxicity in colon cancer cells was assessed by MTT assay. As shown in Fig. 1B, pristimerin treatment caused a dose- and time-dependent inhibition of cell growth in all three tested colon cancer cell lines. Following $48 \mathrm{~h}$ of treatment, the $\mathrm{IC}_{50}$ values of pristimerin in the HCT-116, SW-620 and COLO-205 cells were 1.22 $\pm 0.25,1.04 \pm 0.17$ and $0.84 \pm 0.14 \mu \mathrm{M}$, respectively.

Next, we focused on the effect of pristimerin on colon cancer cell morphology. Untreated HCT-116, SW-620 and COLO-205 cells appeared healthy with clear skeletons, whereas the pristimerin-treated HCT-116 and SW-620 cells were detached and distorted and certain cells became rounded and floated (Fig. 1C). COLO-205 cells treated with pristimerin were distorted with an unclear cytoplasm and skeleton (Fig. 1C). These changes occurred in a dose-dependent manner. Taken together, the MTT assay and morphological data indicated that pristimerin has potent cytotoxic effects on colon cancer cells.

Pristimerin induces $G 1$ phase arrest and modulates cell cyclerelated proteins in colon cancer cells. The effect of pristimerin on the cell cycle was examined by flow cytometric analysis of cellular DNA content. HCT-116, SW-620 and COLO-205 cells were treated with pristimerin for $48 \mathrm{~h}$. As shown in Fig. 2A, pristimerin caused cell cycle transition arrest in the G0/G1 phase and the proportion of cells in the G0/G1 phase 

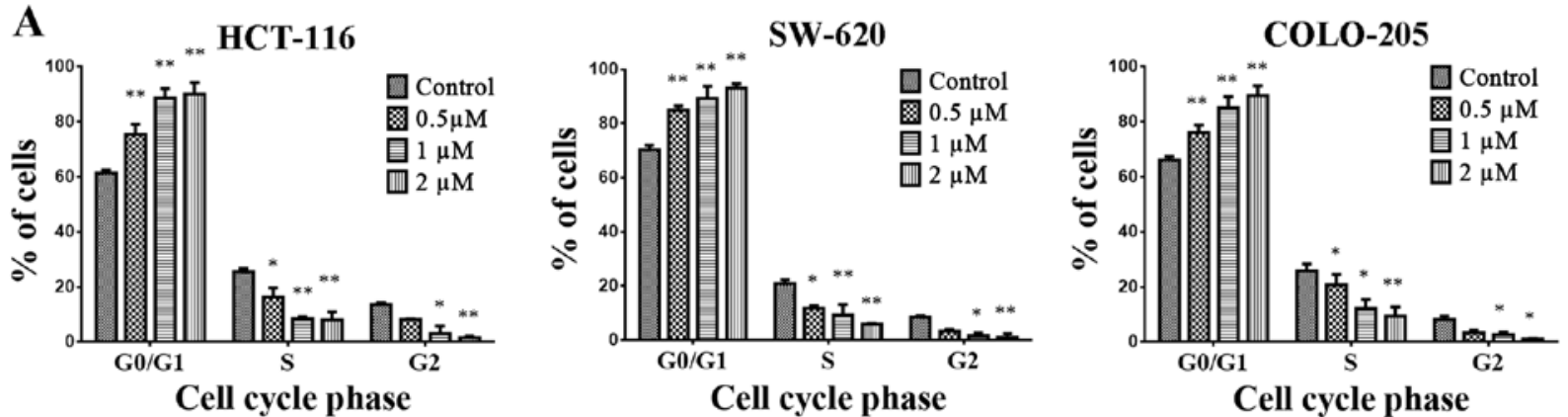

Pristimerin concentration

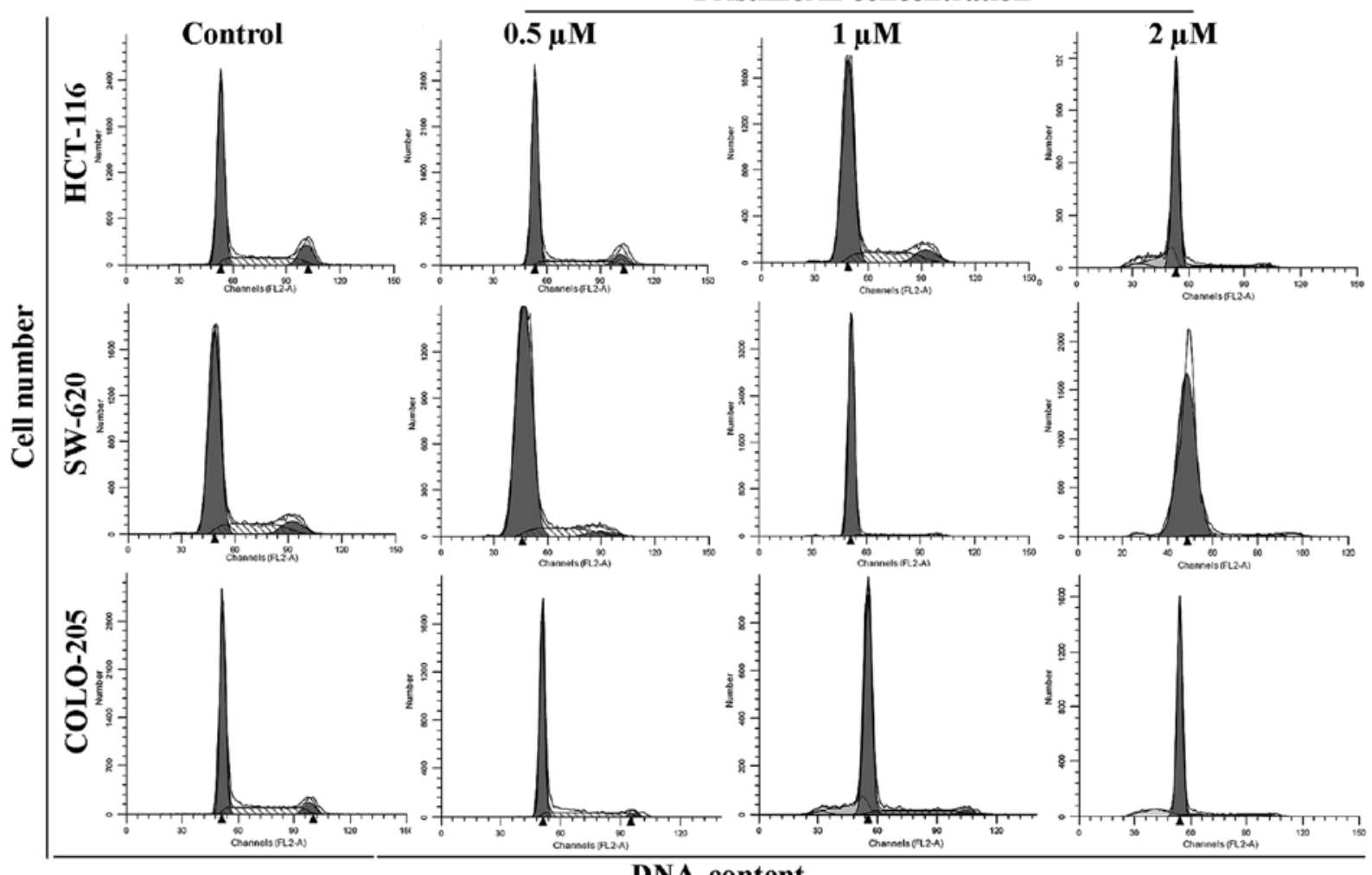

\section{DNA content}

B
HCT-116

Pristimerin conc $(\mu \mathrm{M})$

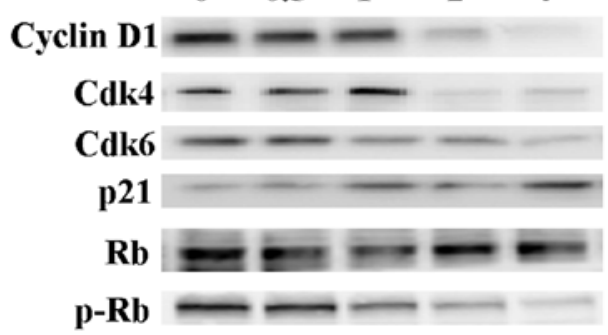

$\beta$-actin

\section{SW-620}

Pristimerin conc $(\mu \mathrm{M})$
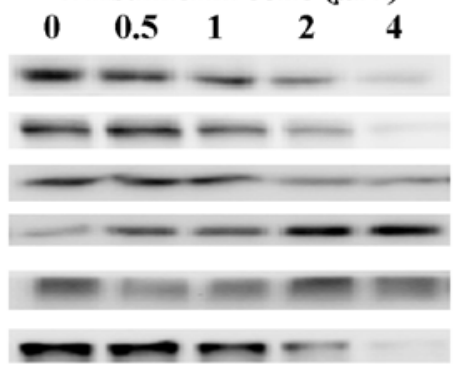

$\longrightarrow$
COLO-205

\section{Pristimerin conc $(\mu \mathrm{M})$}

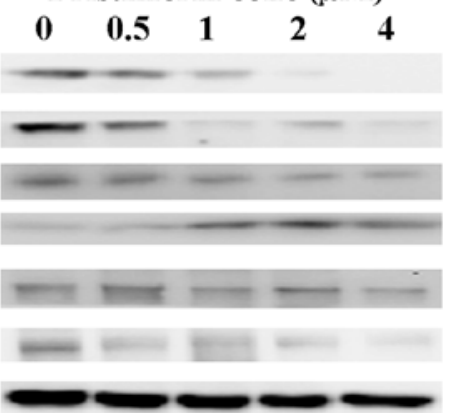

Figure 2. Pristimerin-induced cell cycle arrest. (A) The effects of pristimerin on cell cycle distribution are shown. HCT-116, SW-620 and COLO-205 cells were treated either with pristimerin $(0.5,1$ and $2 \mu \mathrm{M})$ or DMSO (control) for $48 \mathrm{~h}$, and the cells were collected, stained and analyzed for the percentage of cells in different phases of the cell cycle. ${ }^{*} \mathrm{p}<0.05,{ }^{* *} \mathrm{p}<0.01 \mathrm{vs}$. the control. (B) The effects of pristimerin on cell cycle-associated protein expression in the HCT-116, SW-620 and COLO-205 cells which were treated either with pristimerin $(0.5,1,2$ and $4 \mu \mathrm{M})$ or DMSO (control) for $48 \mathrm{~h}$. Total cell lysates were analyzed by western blot analysis (three independent experiments) for G1 cell cycle regulatory proteins (cyclin D1, cdk4, cdk6, p21, Rb and p-Rb). $\beta$-actin was used as the control.

was markedly increased in a concentration-dependent manner. G1 phase cell cycle distribution was $61.2,74.5,86.9$ and $90 \%$
(HCT-116); 70.5, 81.3, 87.9 and 91.2\% (SW-620); 65.8, 74.5, 86.1 and $90.4 \%$ (COLO-205) when the cells were treated with 


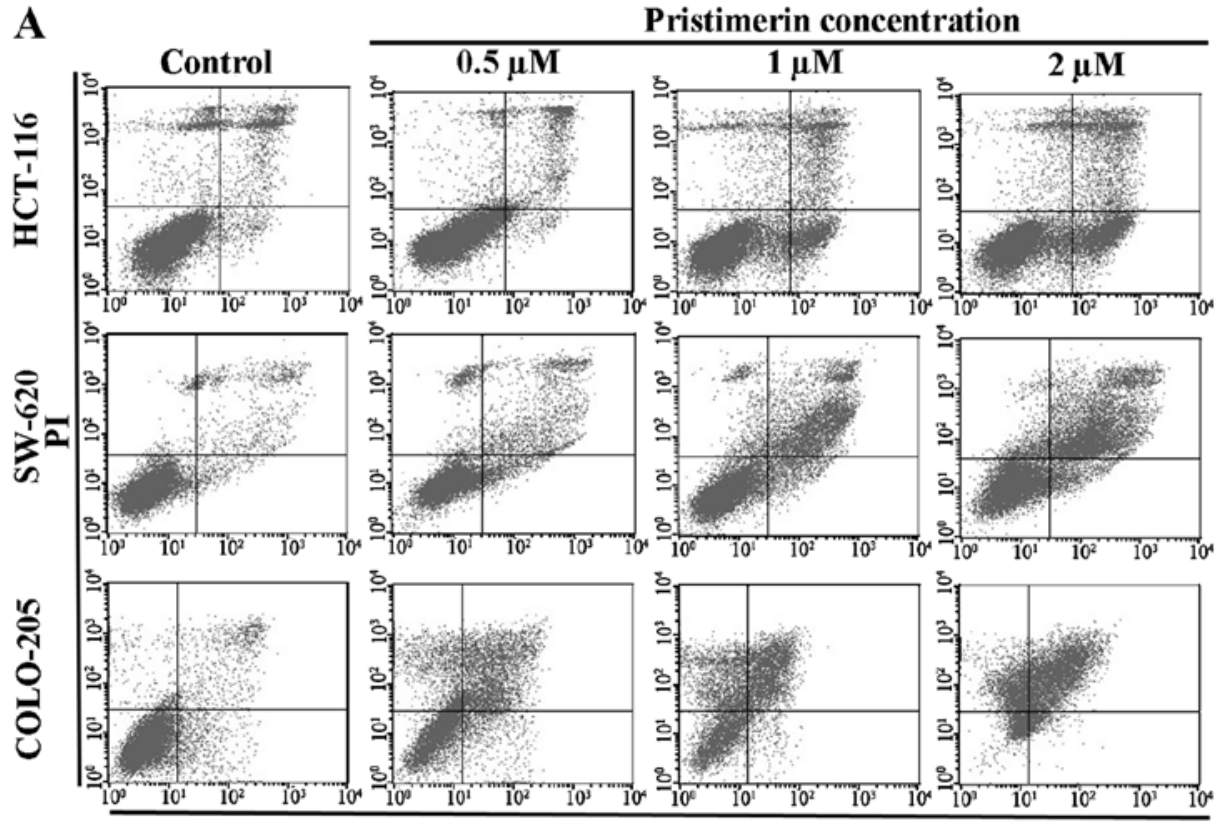

ANNEXIN V

B

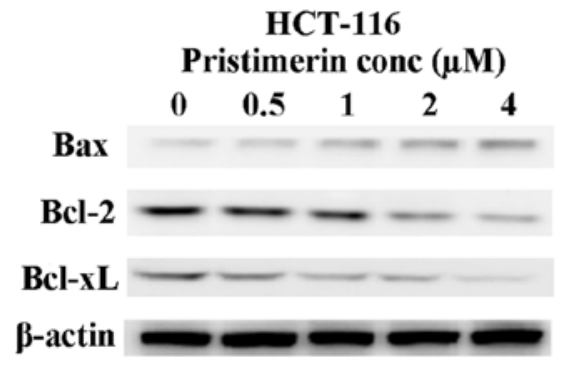

Pristimerin concentration

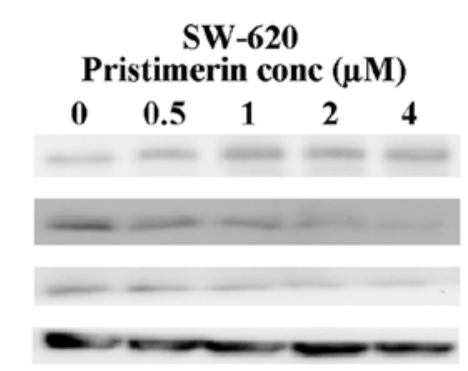

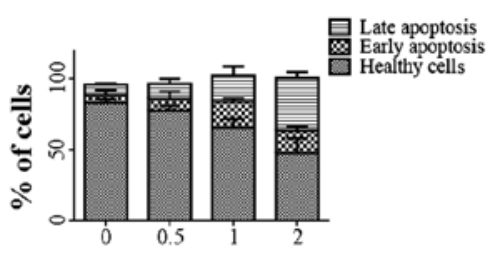

Pristimerin cone $(\mu \mathrm{M})$

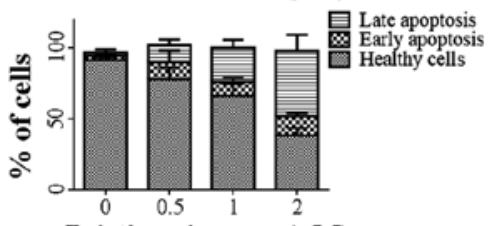

Pristimerin conc $(\mu \mathrm{M})$

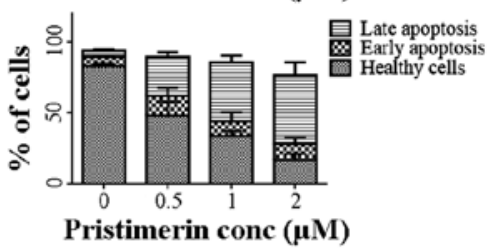

Pristimerin conc $(\mu \mathrm{M})$

COLO-205

Pristimerin conc $(\mu \mathrm{M})$

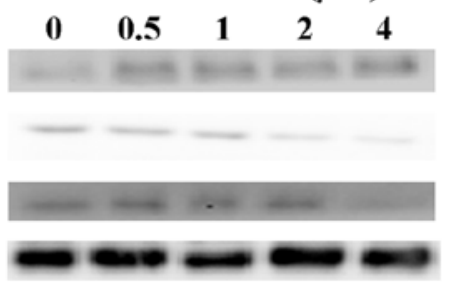

Figure 3. Pristimerin-induced apoptosis in colon cancer cells. (A) Induction of apoptosis as assessed by Annexin V/PI method. HCT-116, SW-620 and COLO-205 cells were treated either with pristimerin $(0.5,1$ or $2 \mu \mathrm{M})$ or DMSO (control) for $48 \mathrm{~h}$ and analyzed using flow cytometry. (B) Effect of pristimerin on Bcl-xL, Bcl-2 and Bax levels. HCT-116, SW-620 and COLO-205 cells were treated either with pristimerin $(0.5,1,2$ and $4 \mu \mathrm{M})$ or DMSO (control) for $48 \mathrm{~h}$. Total cell lysates were analyzed by western blot analysis (three independent experiments). $\beta$-actin was used as the control.

pristimerin at $0,0.5,1$ and $2 \mu \mathrm{M}$, respectively. The increments in the $\mathrm{G} 0 / \mathrm{G} 1$ phases were accompanied by concomitant reduction in the proportion of cells in the $\mathrm{S}$ and $\mathrm{G} 2 / \mathrm{M}$ phases of the cell cycle in the CRC cell lines, with the presence of a small proportion of sub-G1 cells at higher concentrations, which indicated apoptosis. Taken together, these findings suggest that pristimerin-induced G1 arrest in colon cancer cells may be responsible for its growth inhibitory effects.

To understand the mechanism behind pristimerin-induced G1 phase arrest, we investigated the effect of pristimerin on levels of proteins that regulate the progression of cells in the G1 phase including p21, cyclin D1, cdk4, cdk6, retinoblastoma $(\mathrm{Rb})$ and $\mathrm{p}-\mathrm{Rb}$. As shown in Fig. $2 \mathrm{~B}$, the protein level of cyclin D1 was significantly decreased by pristimerin in a concentration-dependent manner in all three colon cancer cell lines. Similarly, a dose-dependent reduction in the expression levels of cdk4 and cdk6 were observed (Fig. 2B). In addition, pristimerin decreased the $\mathrm{p}-\mathrm{Rb}$ level in a dose-dependent manner without change in total Rb protein levels. Furthermore, exposure to pristimerin generated a dose-dependent induction of p21 expression in all three colon cancer cell lines (Fig. 2B). We assumed that the pristimerin modulation of cell cycle regulatory proteins may have contributed to the pristimerinmediated G1 phase arrest in the colon cancer cells.
Pristimerin-induced apoptosis in the colon cancer cells. Pristimerin-induced apoptosis was also studied in colon cancer cells. Cells were treated with varying pristimerin concentrations for $48 \mathrm{~h}$, stained with Annexin V/PI, and subjected to analysis using a flow cytometer. As shown in Fig. 3A, pristimerin treatment of colon cancer cells provoked significant dose-dependent enhancement in both early and late stages of apoptosis. The apoptotic indices were 11.5, 21.4, 39.9 and 55.8\% (HCT-116); and 5.7, 23.4, 36.1 and 64.5\% (SW-620); and $11,35.2,62.7$ and $72.1 \%$ (COLO-205) at $0,0.5,1$ and $2 \mu \mathrm{M}$ concentrations of pristimerin, respectively (Fig. 3A).

To explore how pristimerin induced apoptosis, we studied the action of pristimerin on Bcl-2 family proteins, which play an important role in the regulation of apoptosis, including pro-apoptotic proteins (Bax) and anti-apoptotic proteins (Bcl-2 and $\mathrm{Bcl}-\mathrm{xL}$ ). Western blot analysis showed that pristimerin treatments resulted in a dose-dependent reduction in the levels of anti-apoptotic proteins Bcl-2 and Bcl-xL (Fig. 3B). In contrast, the level of pro-apoptotic protein Bax was significantly increased upon treatment with pristimerin in a dose-dependent manner (Fig. 3B). Thus, pristimerin treatment modulated the Bcl-2 family protein levels in a manner that led to increased ratios of Bax/Bcl-2 and Bax/Bcl-xL, which may have contributed to the observed apoptotic effect of pristimerin. 
A
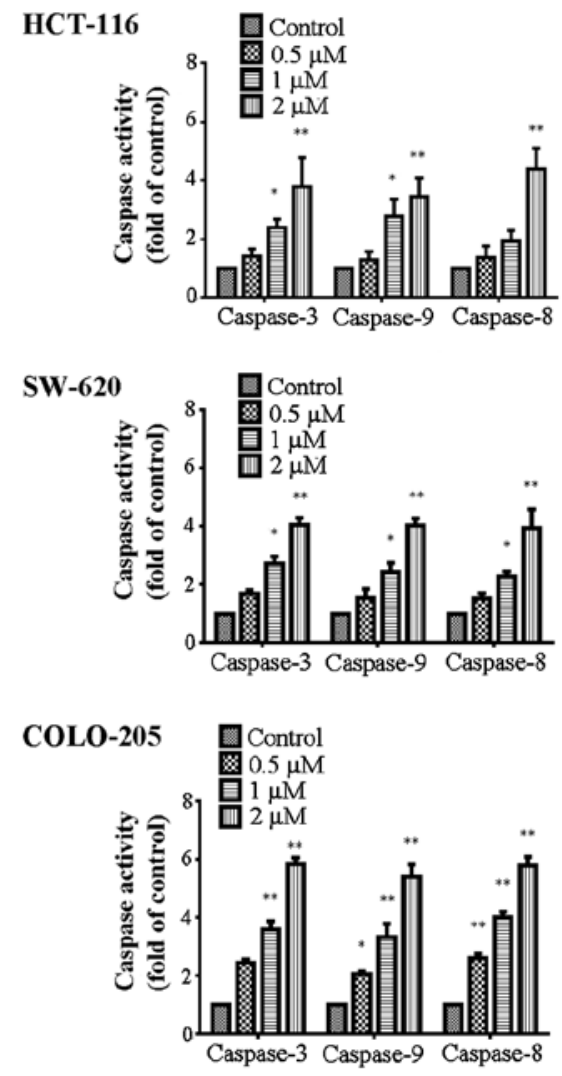

B

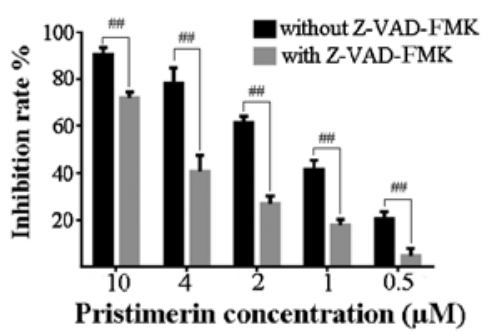

C

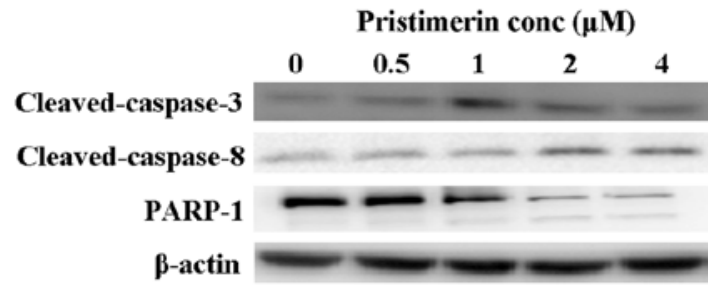

Pristimerin conc $(\mu \mathrm{M})$
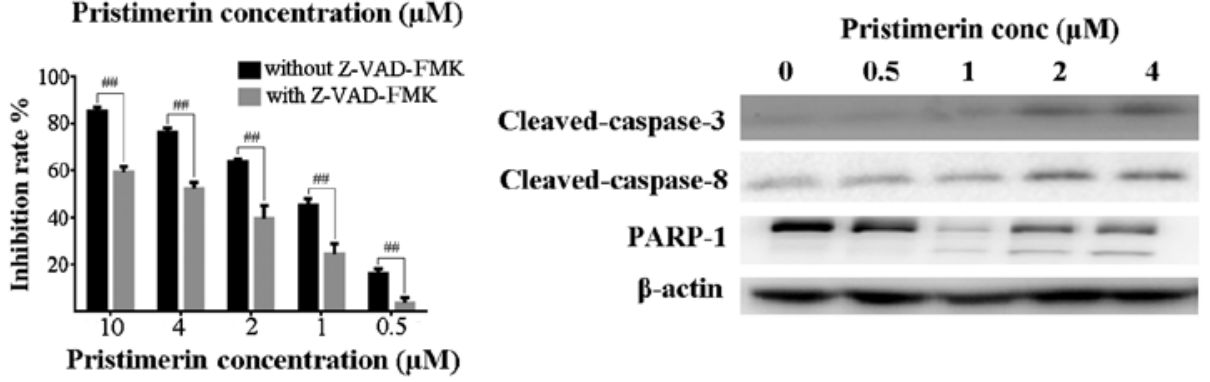

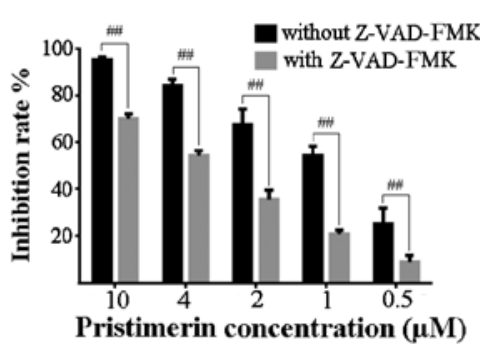

Figure 4. Pristimerin-induced apoptosis via caspase activation in the colon cancer cells. (A) Effect of pristimerin on apoptosis induction as assessed by caspase-3, -8 and -9 activity assays. (B) Effect of pristimerin in both the presence and absence of pan-caspase inhibitor Z-VAD-FMK. Inhibition rate (\%) was determined by MTT assay. Each data are presented as mean \pm SD from three independent experiments. ${ }^{*}$ p $<0.05$, ${ }^{* *}$ p $<0.01$, vs. control. ${ }^{\# \#}$ p $<0.01$ compared to pristimerin treatment in the absence and presence of Z-VAD-FMK. (C) Effect of pristimerin on cleaved-3 and -8 caspase, and PARP-1 protein expression, as assessed by western blotting. $\beta$-actin was used as the control. Western blot analyses represent three independent experiments.

To further understand the effects of pristimerin on cell apoptosis, we studied its effects on caspase- $3,-8$ and -9 activities and PARP-1 activation. Pristimerin treatment caused a dose-dependent enhancement in caspase- $3,-8$ and -9 activities in all the tested cell lines compared with these levels in the control after $48 \mathrm{~h}$ of treatment (Fig. 4A). Pretreatment of cells for $1 \mathrm{~h}$ with $50 \mu \mathrm{l}$ of Z-VAD-FMK, a pan-caspase inhibitor caused a significant reduction in pristimerin cytotoxicity in the colon cancer cells (Fig. 4B). Subsequently, we studied the expression of cleaved caspase- 3 and -8 , and PARP- 1 in all three tested cell lines by western blotting. Pristimerin treatment also increased the expression of cleaved caspase- 3 and -8 , with increased cleavage of PARP-1 in a concentrationdependent manner (Fig. 4C). Taken together, pristimerin induced caspase-dependent apoptotic cell death in the colon cancer cells.

Pristimerin-induced loss of $\Delta \psi m$. Since the intrinsic apoptotic pathway is predominantly triggered by changes in $\Delta \psi \mathrm{m}$, we further investigated the theory that pristimerin treatmentinduced mitochondrial apoptosis was due to the changes in $\Delta \psi \mathrm{m}$. HCT-116, SW-620 and COLO-205 cells treated with pristimerin $(0.5,1$ and $2 \mu \mathrm{M})$ for $48 \mathrm{~h}$ were loaded with $\Delta \Psi \mathrm{m}$ JC-1 probe, and the fluorescent shift in cells was measured by a flow cytometer. There was a significant change in mito- chondrial potential after pristimerin treatment as shown in Fig. 5; pristimerin induced mitochondrial depolerization in a dose-dependent manner.

Pristimerin inhibits EGFR, HER2 activation and related signaling pathways in colon cancer cells. To understand the molecular basis of pristimerin-mediated anticancer activity, we examined the effect of pristimerin on EGFR and HER2 receptors, which are overexpressed in colon cancer (17). In the present study, we demonstrated that pristimerin exhibited anticancer activity against HCT-116 (EGFR strong), COLO-205 (EGFR weak) and SW-620 (EGFR negative), while all three cell lines showed detectable HER2 levels. Our results showed that pristimerin inhibited EGFR phosphorylation in a dosedependent manner in the HCT-116 and COLO-205 cells, and inhibited HER2 phosphorylation in the three tested cell lines in a dose-dependent manner, with insignificant change in total EGFR and HER-2 protein expression (Fig. 6). Next, we evaluated whether the pristimerin-dependent reduction in EGFR and HER 2 activities was associated with reduced downstream kinase activity including mitogen-activated protein kinase (MAPK), phosphatidylinositol 3-kinase (PI3K)/Akt/mTOR and NF- $\mathrm{KB}$ signaling pathways (18). We found that pristimerin significantly suppressed phosphorylation of ERK1/2, Akt, mTOR and NF-kB in a dose-dependent manner (Fig. 6), with 

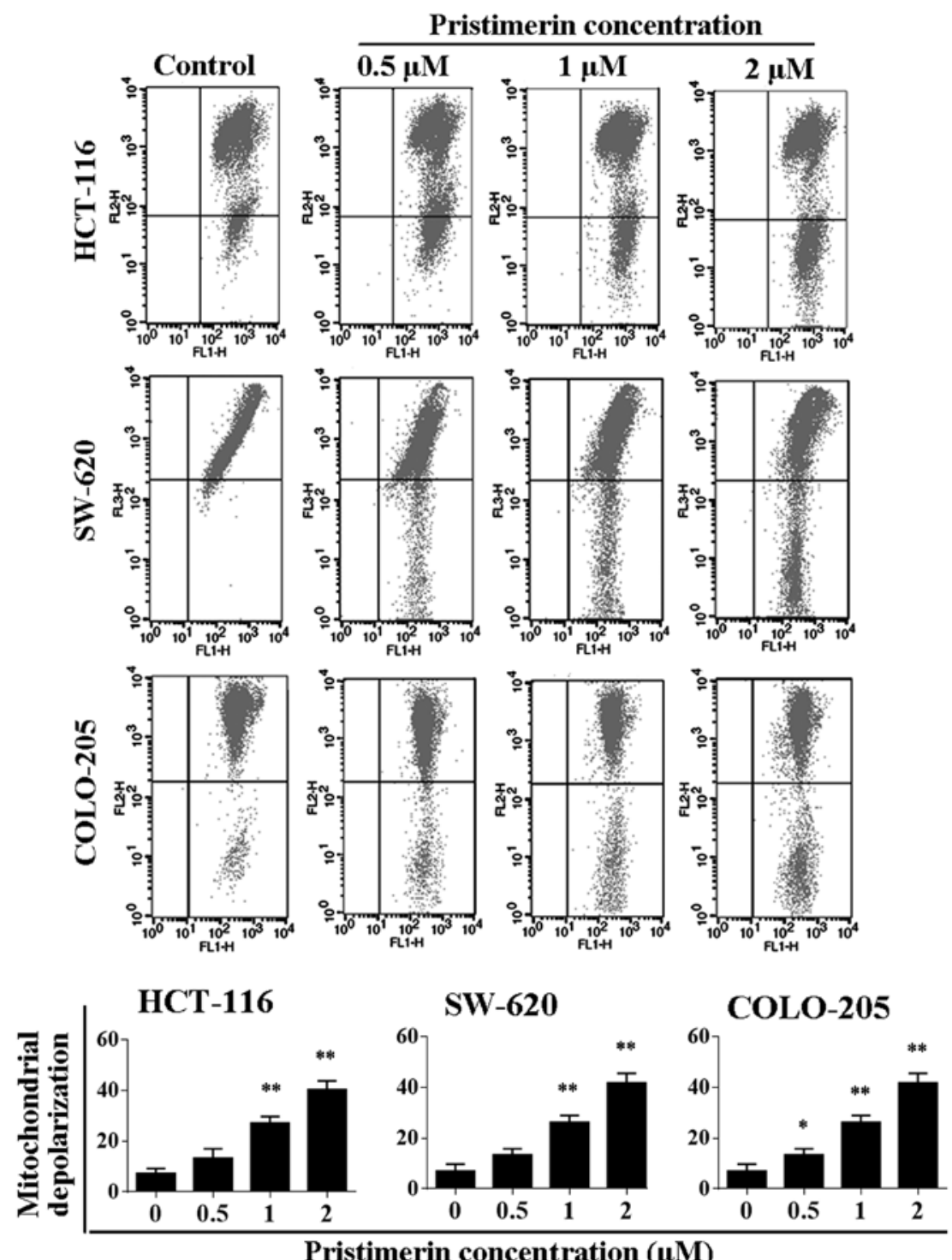

Figure 5. Pristimerin-induced mitochondrial depolarization in colon cancer cells. HCT-116, SW-620 and COLO-205 cells were treated with pristimerin $(0.5,1$ and $2 \mu \mathrm{M}$ ) or DMSO (control) for $48 \mathrm{~h}$. Cells were collected and stained with mitochondrial potential sensor JC-1 and analyzed for mitochondrial membrane potential loss. Data are represented as the mean \pm SD from three independent experiments. ${ }^{*} \mathrm{p}<0.05,{ }^{* *} \mathrm{p}<0.01$ vs. the control.

no effect on total protein expression. The results suggest that pristimerin exerts its anticancer effects through both inhibition of EGFR and HER-2 activation on colon cancer cell surfaces and suppression of downstream ERK1/2, Akt, mTOR and NF- $\mathrm{BB}$ signaling pathways.

\section{Discussion}

Plants are important sources of biologically active natural products which have become more important in anticancer drug development as they are more tolerable to the human body (5). Pristimerin is a natural triterpenoid that has been shown to possess a variety of biological activities. It has been reported to exhibit anticancer activity against different human cancer cells (19). In the present study, we examined the pristimerin cytotoxicity against three colon cancer cell lines for the first time. Our experimental results showed that pristimerin displayed dose- and time-dependent potent cytotoxicity in colon cancer cells. The cytotoxic effects were further confirmed by studied morphological changes induced by pristimerin on human colon cancer cells.

Persistence of cell cycle progression in cancer cells is regarded as one of the hallmarks of cancer (20). Therefore, it is considered as a target for the treatment of human malignancies (21). Pristimerin was found to induce cell cycle arrest in a variety of human cancer cell lines, including pancreatic cancer (9) and myeloma tumors (15). Our results revealed that pristimerin treatment of colon cancer cells induced a dose-dependent arrest of cells in the G1 phase. Cell cycle progression is highly controlled by many processes involved in activation of cyclin-dependent kinases (CDKs), which are paired with the cyclins (e.g. cyclins A, B, E, D1-3) (22). Activation of CDKs by cyclins leads to phosphorylation of $\mathrm{Rb}$ and leads to diminished binding of $\mathrm{Rb}$ to the $\mathrm{E} 2 \mathrm{~F}$ transcription factor, which enhances transcription of various genes necessary for cell cycle progression (23). The activities of CDKs are antagonized by CDK inhibitors, such as p27 and p21, which directly interact with CDKs (24). In order to investigate the 


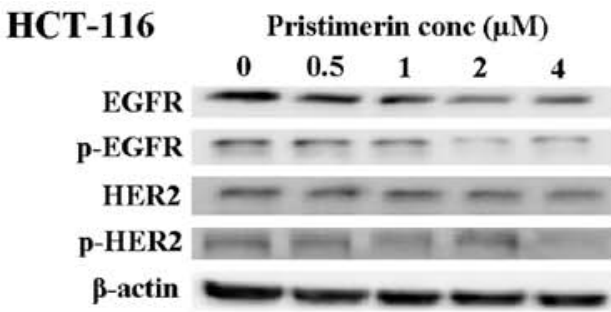

SW-620
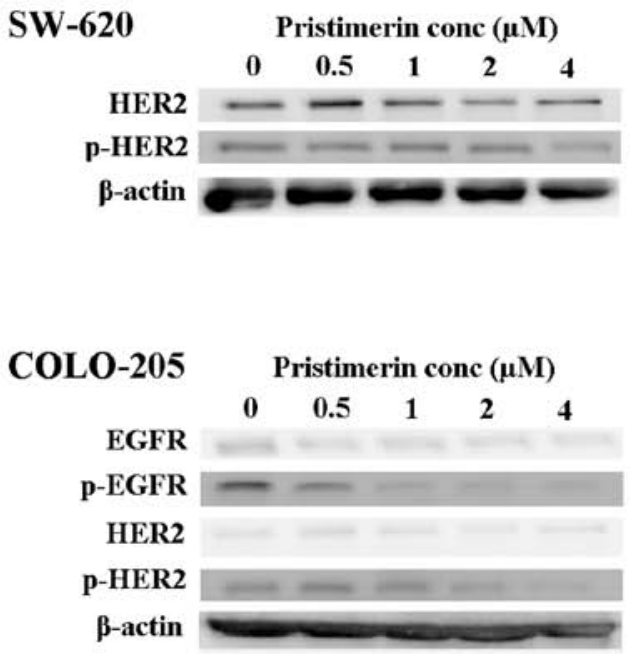
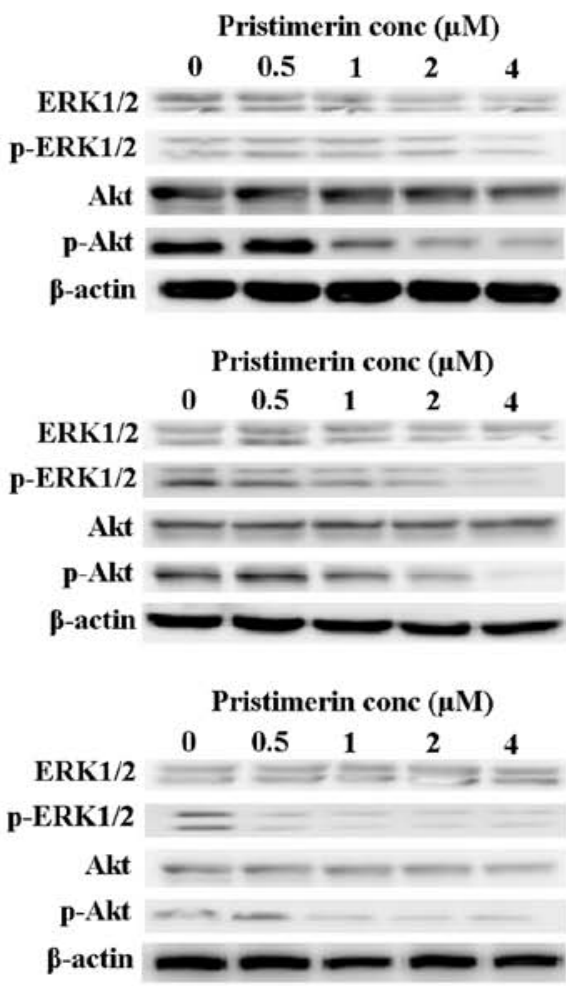

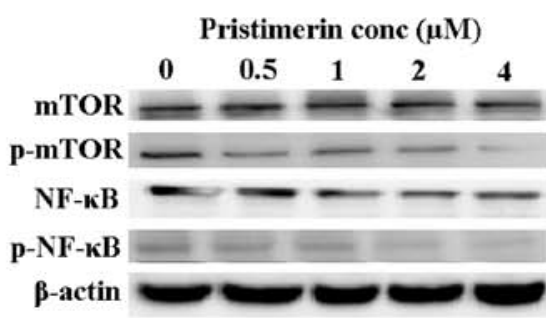

Pristimerin conc $(\mu \mathrm{M})$

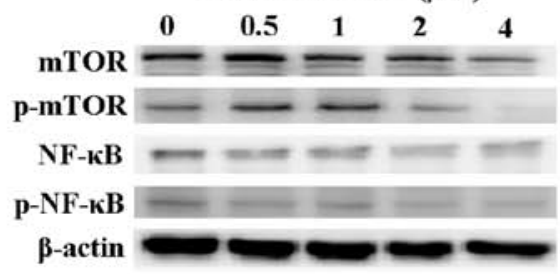

Pristimerin conc $(\mu \mathrm{M})$

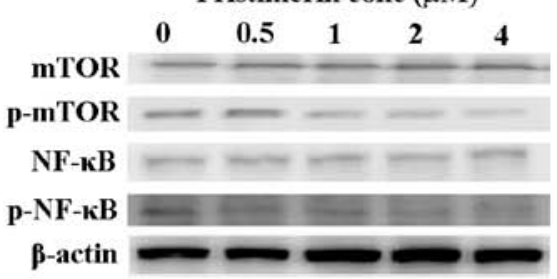

Figure 6. Pristimerin inhibited EGFR, HER2 and related downstream signaling proteins in colon cancer cells. HCT-116, SW-620 and COLO-205 cells were treated either with pristimerin $(0.5,1$ and $2 \mu \mathrm{M})$ or DMSO (control) for $48 \mathrm{~h}$. Total cell lysates were analyzed by western blot analysis (three independent experiments) for EFGR, p-EGFR, HER2 and p-HER2. In addition, downstream targets (ERK1/2, p-ERK1/2, Akt, p-Akt, mTOR, p-mTOR, NF- $\kappa B$ and $\mathrm{p}-\mathrm{NF}-\kappa \mathrm{B})$ also were detected. $\beta$-actin was used as control.

mechanism underlying pristimerin-induced G1 phase cell cycle arrest, cell cycle regulatory proteins, CDKs and CDK inhibitors were analyzed using western blot analysis. Our results showed that pristimerin upregulated p21 expression, along with decreased cyclin D1, Cdk-4 and Cdk-6 expression. In addition, pristimerin decreased phosphorylated Rb expression, which led to G1 phase arrest. These findings suggest that pristimerin-induced G1 phase cell cycle arrest may be mediated through the cyclin-CDK checkpoint.

Apoptotic resistance is another important hallmark of cancer cells (20). Thus, activation of apoptotic pathways is a key mechanism by which anticancer drugs kill tumor cells (25). Apoptosis is a highly regulated and organized cell death process. Two major pathways of apoptosis, depending on different apoptotic stimuli, are the mitochondrial pathway (intrinsic) and the death receptor pathway (extrinsic) (26). In the present study, we observed that pristimerin induced apoptotic cell death in a dose-dependent manner, which was confirmed by Annexin V/PI double staining, confirming membrane alterations related to apoptosis in colon cancer cells. Moreover, our caspase activity assay showed that pristimerin was able to induce caspase-dependent apoptosis in human colon cancer cells in a dose-dependent manner. This caspase-dependent apoptosis was further explored by the presence of pan-caspase inhibitor (Z-VAD-FMK), which reduced the cytotoxic activity of pristimerin. We found that pristimerin induced apoptosis via the cell death receptor pathway (extrinsic) by activating caspase- 8 and -3 . These results were in line with a previous study on human breast cancer cells (27). Furthermore, pristimerin showed activation of caspase- 9 and -3 , which are modulators of the mitochondrial pathway (intrinsic) (28). The activation of caspases are crucial steps in apoptotic cell death, which consequently induce PARP-1 cleavage, another marker of cell apoptosis (29).

Mitochondria have been shown to play a central role in the apoptotic process, through both intrinsic and extrinsic pathways. Mitochondrial membrane permeability is one of the most important features of the mitochondria, and permeability change, mainly through permeability transition pores, leads to pro-apoptotic factor release, loss of mitochondrial membrane potential $(\Delta \psi \mathrm{m})$ and cell death (30). Our results showed that pristimerin induced conformational changes of mitochondria, followed by redistribution of pro-apoptotic factors and $\Delta \Psi \mathrm{m}$ loss in a dose-dependent manner. Bcl-2, Bcl-xL and Bax have been implicated as major regulators of the role of mitochondrial in apoptosis (31). Pro-apoptotic Bcl-2 members bind to the mitochondrial outer membrane and block cytochrome $c$ efflux. In apoptosis, Bax translocates from the cytosol to the mitochondria where it increases the efflux of cytochrome $c$ through the mitochondrial outer membrane (32). Thus, changes in anti-apoptotic and pro-apoptotic Bcl-2 family proteins are critical for the induction of apoptosis (33). Pristimerin treatment of colon cancer cells increased Bax protein expression and decreased both Bcl-2 and Bcl-xL expression thereby increasing the $\mathrm{Bax} / \mathrm{Bcl}-2$ and $\mathrm{Bax} / \mathrm{Bcl}-\mathrm{xL}$ ratios leading to apoptosis. Collectively, our results revealed that pristimerin induced both intrinsic and extrinsic apoptotic pathways in the colon cancer cell lines.

Overexpression of growth factors and their receptors (EGFR and HER2) play a vital role in the tumorigenesis of 
colorectal carcinoma, which leads to constitutive activation of tyrosine kinase activity of EGFR and related downstream signaling pathways $(17,34)$. Therefore, agents that target EGFR and/or HER2 receptor show anticancer activity against CRC (35). Our results showed that the tested colon cancer cell lines had different expression patterns of growth factor receptors, as previously reported (36). Pristimerin inhibited EGFR and HER2 activation in a dose-dependent manner. The same results were previously reported in glioma tumors (13) and breast cancer cells (27). Upregulation and activation of EGFR and HER-2 were found to lead to the activation of multiple intracellular signaling cascades that are responsible for tumorigenesis of colon cancer including MAPK, PI3K/ Akt $/ m-T O R$ and NF- $\kappa$ B signaling pathways $(18,37)$. The downstream MAPK/ERK signaling pathway plays an important role in cell proliferation and apoptosis regulation (38). Our results showed that pristimerin blocked ERK1/2 phosphorylation. The same results were obtained in hepatic (10) and breast cancer cells (39). PI3K/AKT is another downstream signaling pathway of EFGR/HER2 that controls cell proliferation, survival, apoptosis and malignant transformation (40). Our results illustrated that pristimerin reduced Akt activation as a consequence of EFGR/HER2 inhibition. Anti-apoptotic/ pro-survival $\mathrm{NF}-\kappa \mathrm{B}$ and $\mathrm{mTOR}$ signaling pathways are downstream targets of activated PI3K/AKT, which controls cell growth, survival, division and motility $(41,42)$. Pristimerin also blocked both phosphorylation and activation of these pathways. Other researchers found the same results, in which pristimerin inhibited pro-survival Akt/NF- $\mathrm{B} / \mathrm{m}-\mathrm{TOR}$ signaling proteins in pancreatic cancer cells (43). Pristimerin inhibited the expression of p-ERK, p-Akt, p-NF- $\mathrm{B}$ and p-mTOR in colon cancer cells, which indicated that inhibition of those pro-survival signaling proteins was essential for pristimerin-induced apoptosis.

In conclusion, our findings revealed that pristimerin induced cell cycle arrest and apoptosis in human colon cancer cells HCT-116, SW-620 and COLO-205 through cyclin-CDK, mitochondrial dysfunction and caspase-dependent mechanisms. The mechanisms may involve inhibition of phosphorylated EGFR and HER 2 expression, and subsequently caused inhibition of related downstream kinases. These findings suggest that pristimerin may be a novel candidate for colon cancer therapy.

\section{Acknowledgements}

The present study was funded by the Priority Academic Program Development of Jiangsu Higher Education Institutions (PAPD), also supported by the fundamental research funds for the central universities (YD2014SK0002). The present study was also partially supported by the 111 Project (111-2-07), and the National 12th Five-Year Plan: The 'Major Scientific and Technological Special Project for Significant New Drug Creation' Project (no. 2012ZX09504001-001).

\section{References}

1. Tenesa A and Dunlop MG: New insights into the aetiology of colorectal cancer from genome-wide association studies. Nat Rev Genet 10: 353-358, 2009.

2. Siegel R, Ma J, Zou Z and Jemal A: Cancer statistics, 2014. CA Cancer J Clin 64: 9-29, 2014.
3. Wood LD, Parsons DW, Jones S, Lin J, Sjöblom T, Leary RJ, Shen D, Boca SM, Barber T, Ptak J, et al: The genomic landscapes of human breast and colorectal cancers. Science 318: 1108-1113, 2007.

4. Cancer Genome Atlas Network: Comprehensive molecular characterization of human colon and rectal cancer. Nature 487: 330-337, 2012.

5. Newman DJ, Cragg GM and Snader KM: Natural products as sources of new drugs over the period 1981-2002. J Nat Prod 66: 1022-1037, 2003.

6. Brinker AM, Ma J, Lipsky PE and Raskin I: Medicinal chemistry and pharmacology of genus Tripterygium (Celastraceae). Phytochemistry 68: 732-766, 2007.

7. Dirsch VM, Kiemer AK, Wagner H and Vollmar AM: The triterpenoid quinonemethide pristimerin inhibits induction of inducible nitric oxide synthase in murine macrophages. Eur J Pharmacol 336: 211-217, 1997.

8. Wu CC, Chan ML, Chen WY, Tsai CY, Chang FR and Wu YC: Pristimerin induces caspase-dependent apoptosis in MDA-MB231 cells via direct effects on mitochondria. Mol Cancer Ther 4: 1277-1285, 2005

9. Wang Y, Zhou Y, Zhou H, Jia G, Liu J, Han B, Cheng Z, Jiang H, Pan S and Sun B: Pristimerin causes G1 arrest, induces apoptosis, and enhances the chemosensitivity to gemcitabine in pancreatic cancer cells. PLoS One 7: e43826, 2012.

10. Guo Y, Zhang W, Yan YY, Ma CG, Wang X, Wang C and Zhao JL: Triterpenoid pristimerin induced HepG2 cells apoptosis through ROS-mediated mitochondrial dysfunction. J BUON 18: 477-485, 2013.

11. Byun JY, Kim MJ, Eum DY, Yoon CH, Seo WD, Park KH, Hyun JW, Lee YS, Lee JS, Yoon MY, et al: Reactive oxygen species-dependent activation of Bax and poly(ADP-ribose) polymerase-1 is required for mitochondrial cell death induced by triterpenoid pristimerin in human cervical cancer cells. Mol Pharmacol 76: 734-744, 2009.

12. Liu YB, Gao X, Deeb D, Brigolin C, Zhang Y, Shaw J, Pindolia K and Gautam SC: Ubiquitin-proteasomal degradation of antiapoptotic survivin facilitates induction of apoptosis in prostate cancer cells by pristimerin. Int J Oncol 45: 1735-1741, 2014.

13. Yan YY, Bai JP, Xie Y, Yu JZ and Ma CG: The triterpenoid pristimerin induces U87 glioma cell apoptosis through reactive oxygen species-mediated mitochondrial dysfunction. Oncol Lett 5: 242-248, 2013.

14. Yang H, Landis-Piwowar KR, Lu D, Yuan P, Li L, Reddy GP, Yuan X and Dou QP: Pristimerin induces apoptosis by targeting the proteasome in prostate cancer cells. J Cell Biochem 103: 234-244, 2008

15. Tiedemann RE, Schmidt J, Keats JJ, Shi CX, Zhu YX, Palmer SE, Mao X, Schimmer AD and Stewart AK: Identification of a potent natural triterpenoid inhibitor of proteosome chymotrypsin-like activity and NF-kappaB with antimyeloma activity in vitro and in vivo. Blood 113: 4027-4037, 2009.

16. Liu C, Yu K, Shi X, Wang J, Lam PK, Wu RS and Zhou B: Induction of oxidative stress and apoptosis by PFOS and PFOA in primary cultured hepatocytes of freshwater tilapia (Oreochromis niloticus). Aquat Toxicol 82: 135-143, 2007.

17. Milano G, Etienne-Grimaldi MC, Dahan L, Francoual M, Spano JP, Benchimol D, Chazal M, Letoublon C, André T, Gilly FN, et al: Epidermal growth factor receptor (EGFR) status and K-Ras mutations in colorectal cancer. Ann Oncol 19: 2033-2038, 2008.

18. Spano JP, Fagard R, Soria JC, Rixe O, Khayat D and Milano G: Epidermal growth factor receptor signaling in colorectal cancer: Preclinical data and therapeutic perspectives. Ann Oncol 16: 189-194, 2005.

19. Costa PM, Ferreira PM, Bolzani VS, Furlan M, de Freitas Formenton Macedo Dos Santos VA, Corsino J, de Moraes MO, Costa-Lotufo LV, Montenegro RC and Pessoa C: Antiproliferative activity of pristimerin isolated from Maytenus ilicifolia (Celastraceae) in human HL-60 cells. Toxicol In Vitro 22: 854-863, 2008

20. Hanahan D and Weinberg RA: Hallmarks of cancer: The next generation. Cell 144: 646-674, 2011.

21. Call JA, Eckhardt SG and Camidge DR: Targeted manipulation of apoptosis in cancer treatment. Lancet Oncol 9: 1002-1011, 2008.

22. Morgan DO: Cyclin-dependent kinases: Engines, clocks, and microprocessors. Annu Rev Cell Dev Biol 13: 261-291, 1997.

23. Bartek J, Bartkova J and Lukas J: The retinoblastoma protein pathway and the restriction point. Curr Opin Cell Biol 8: 805-814, 1996. 
24. Coqueret O: New roles for $\mathrm{p} 21$ and p27 cell-cycle inhibitors: A function for each cell compartment? Trends Cell Biol 13: 65-70, 2003.

25. Debatin KM: Activation of apoptosis pathways by anticancer drugs. Adv Exp Med Biol 457: 237-244, 1999.

26. Hengartner MO: The biochemistry of apoptosis. Nature 407: 770-776, 2000.

27. Lee JS, Yoon IS, Lee MS, Cha EY, Thuong PT, Diep TT and Kim JR: Anticancer activity of pristimerin in epidermal growth factor receptor 2-positive SKBR3 human breast cancer cells. Biol Pharm Bull 36: 316-325, 2013

28. Yasuda Y, Saito M, Yamamura T, Yaguchi T and Nishizaki T: Extracellular adenosine induces apoptosis in Caco-2 human colonic cancer cells by activating caspase- $9 /-3$ via $\mathrm{A}_{2 \mathrm{a}}$ adenosine receptors. J Gastroenterol 44: 56-65, 2009.

29. Yang Y, Zhao S and Song J: Caspase-dependent apoptosis andindependent poly(ADP-ribose) polymerase cleavage induced by transforming growth factor $\beta 1$. Int J Biochem Cell Biol 36: 223-234, 2004

30. Jacotot E, Costantini P, Laboureau E, Zamzami N, Susin SA and Kroemer G: Mitochondrial membrane permeabilization during the apoptotic process. Ann NY Acad Sci 887: 18-30, 1999.

31. Brunelle JK and Letai A: Control of mitochondrial apoptosis by the Bcl-2 family. J Cell Sci 122: 437-441, 2009.

32. Scorrano L and Korsmeyer SJ: Mechanisms of cytochrome $c$ release by proapoptotic BCL-2 family members. Biochem Biophys Res Commun 304: 437-444, 2003.

33. Llambi F and Green DR: Apoptosis and oncogenesis: Give and take in the BCL-2 family. Curr Opin Genet Dev 21: 12-20, 2011.

34. Porebska I, Harlozińska A and Bojarowski T: Expression of the tyrosine kinase activity growth factor receptors (EGFR, ERB B2, ERB B3) in colorectal adenocarcinomas and adenomas. Tumour Biol 21: 105-115, 2000.

35. Raymond E, Faivre S and Armand JP: Epidermal growth factor receptor tyrosine kinase as a target for anticancer therapy. Drugs 60 (Suppl 1): S15-S42, 2000.
36. Balin-Gauthier D, Delord JP, Rochaix P, Mallard V, Thomas F, Hennebelle I, Bugat R, Canal P and Allal C: In vivo and in vitro antitumor activity of oxaliplatin in combination with cetuximab in human colorectal tumor cell lines expressing different level of EGFR. Cancer Chemother Pharmacol 57: 709-718, 2006.

37. Seshacharyulu P, Ponnusamy MP, Haridas D, Jain M, Ganti AK and Batra SK: Targeting the EGFR signaling pathway in cancer therapy. Expert Opin Ther Targets 16: 15-31, 2012.

38. McCubrey JA, Steelman LS, Chappell WH, Abrams SL, Wong EW, Chang F, Lehmann B, Terrian DM, Milella M, Tafuri A, et al: Roles of the Raf/MEK/ERK pathway in cell growth, malignant transformation and drug resistance. Biochim Biophys Acta 1773: 1263-1284, 2007.

39. Mu X, Shi W, Sun L, Li H, Jiang Z and Zhang L: Pristimerin, a triterpenoid, inhibits tumor angiogenesis by targeting VEGFR2 activation. Molecules 17: 6854-6868, 2012.

40. Vivanco I and Sawyers CL: The phosphatidylinositol 3-Kinase AKT pathway in human cancer. Nat Rev Cancer 2: 489-501, 2002.

41. Sekulić A, Hudson CC, Homme JL, Yin P, Otterness DM, Karnitz LM and Abraham RT: A direct linkage between the phosphoinositide 3-kinase-AKT signaling pathway and the mammalian target of rapamycin in mitogen-stimulated and transformed cells. Cancer Res 60: 3504-3513, 2000.

42. Mayo MW and Baldwin AS: The transcription factor NF-kappaB: Control of oncogenesis and cancer therapy resistance. Biochim Biophys Acta 1470: M55-M62, 2000.

43. Deeb D, Gao X, Liu YB, Pindolia K and Gautam SC: Pristimerin, a quinonemethide triterpenoid, induces apoptosis in pancreatic cancer cells through the inhibition of pro-survival Akt/NF- $\kappa \mathrm{B} /$ mTOR signaling proteins and anti-apoptotic Bcl-2. Int $\mathrm{J}$ Oncol 44: 1707-1715, 2014. 